\title{
Nonlinear phononic crystals based on chains of disks alternating with toroidal structures
}

\author{
A. Spadoni, ${ }^{1}$ C. Daraio, ${ }^{1, a)}$ W. Hurst, ${ }^{2}$ and M. Brown ${ }^{2}$ \\ ${ }^{1}$ Engineering and Applied Science, California Institute of Technology, Pasadena, California 91125, USA \\ ${ }^{2}$ Aerospace Research Laboratories, Northrop Grumman Aerospace Systems, Redondo Beach, \\ California 90278 USA
}

(Received 26 October 2010; accepted 23 February 2011; published online 18 April 2011)

\begin{abstract}
We study experimentally the acoustic response of a load-bearing, phononic crystal composed of alternating steel disks, and polytetrafluoroethylene o-rings under precompression. The crystal allows for axial, rocking, and shear-polarized wavemodes when excited by a broad-band signal applied off-axis. Finite element analysis is employed to determine the system's wave modes. The nonlinear interaction between disks and o-rings supports a dynamic response that is tunable with variations in static precompression, leading to controllable frequency shifts in a large band gap. A modal analysis reveals that four of the six principal wave modes are susceptible to external precompression while two modes are not. () 2011 American Institute of Physics. [doi:10.1063/1.3567753]
\end{abstract}

The periodic modulation of geometric and material properties in phononic crystals (PCs) results in acoustic impedance mismatch, which in turn leads to an effective medium with a nonlinear dispersion relation. PCs can be designed to inhibit the propagation of waves within certain frequencies known as band gaps, ${ }^{1}$ in which signals decay exponentially. Pass bands in PCs, additionally, can enable unique behaviors like negative refraction, ${ }^{2}$ used in the past to focus sound. ${ }^{3}$

In classical PCs, band gaps, and negative refraction occur at fixed frequencies dictated by geometric and material properties. Certain PCs designs also inhibit loadbearing capabilities. ${ }^{2}$ This characteristic and the fixed operational range, limit the employment of linear PCs in practical applications. Researchers are now investigating tunable PCs with band gaps controlled by external fields: temperature-sensitive, ${ }^{4}$ magneto-sensitive, ${ }^{5}$ and electrorheological materials ${ }^{5}$ are some examples. Strain-induced geometric transformations of voids in elastomeric domains allow the extinction of certain band gaps and the introduction of new ones. ${ }^{6}$ Static precompression has been used to control the dynamic response of chains of spherical particles. ${ }^{7,8}$

In this letter, we describe the behavior of a PC composed of alternating linear elastic o-rings and disks excited by broad-band vibrations. The o-rings, made of polytetrafluoroethylene (PTFE), have a cross-sectional diameter $d_{c}=4.8$, and a ring diameter $d_{0}=97 \mathrm{~mm}$. The steel disks have a diameter $d_{s}=114 \mathrm{~mm}$ and thickness $h_{s}=13 \mathrm{~mm}$. Material properties for PTFE are: density $\rho_{c}=2150 \mathrm{Kg} / \mathrm{m}^{3}$, stiffness $E_{c}=0.5 \mathrm{GPa}$, and Poisson's ratio $\nu_{c}=0.46$; for steel $\rho_{s}$ $=8000 \mathrm{Kg} / \mathrm{m}^{3}, E_{s}=200 \mathrm{GPa}$, and $\nu_{s}=0.33$. The contact interaction between o-ring and disks [Figs. 1(a) and 1(b)] is geometrically nonlinear and it is sensitive to external precompression..$^{9,10}$

We tested experimentally a system composed of four teflon o-rings and five steel disks [Fig. 1(a)]. A heavy steel plate and brackets emulated fixed boundary conditions. The opposite side was connected to a shaker (LDS $10 \mathrm{lb}$ ) to excite broad-band vibrations off-axis inducing both axial and rocking modes. A thin layer of glue ensured alignment be-

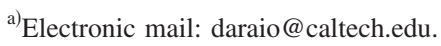

tween the disks and the o-rings, and at the wall. Small holes in the steel disks accommodated a cable-and-pulley system used to apply static precompression. The initial strain state of the crystal determined the effective interaction stiffness between the elements in the chain. Variation in the interaction stiffness allowed tuning the position of the acoustic band gaps in the system's dispersion relation, provided dynamic signals had amplitude lower or comparable to the applied static precompression. This tunability could be exploited even during operation, allowing for dynamic control of the system's response. In the following analyses, we neglect dissipation originating from PTFE or glue layers given that the response is dominated by a significant band gap.

We also developed a finite element (FE) model to characterize wave modes excited in the system. The employed FE discretization of the representative volume element (RVE) is composed of a single o-ring and two steel disks of half thickness $h_{s} / 2$, and it is discretized with eight-node solid elements [Fig. 1(c)]. Dispersion relations for a RVE of $z$-dimension $L$ were obtained imposing periodic conditions on displacements of the RVE extrema $\underline{u}_{\text {top }}=[I] e^{i \mu L} \underline{u}_{\text {bot }}$ in ad-

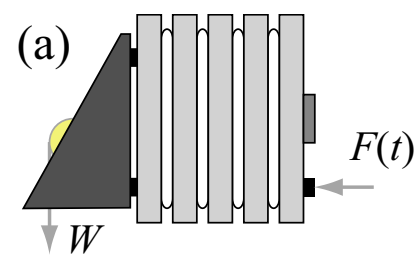

(b)

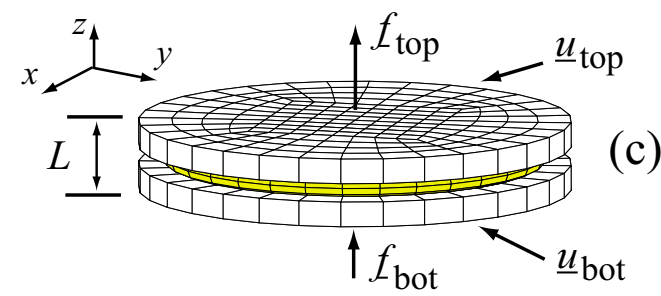

FIG. 1. (Color online) (a) Experimental setup composed of alternating steel disks and teflon o-rings (b) clamped at one end and precompressed by a cable and pulley system with load $W$. A shaker imparted dynamic excitation $F(t)$ and force sensors recorded the motion (solid black blocks). (c) Finiteelement discretization of the RVE. $F(t)$ is the input force at the boundary. 

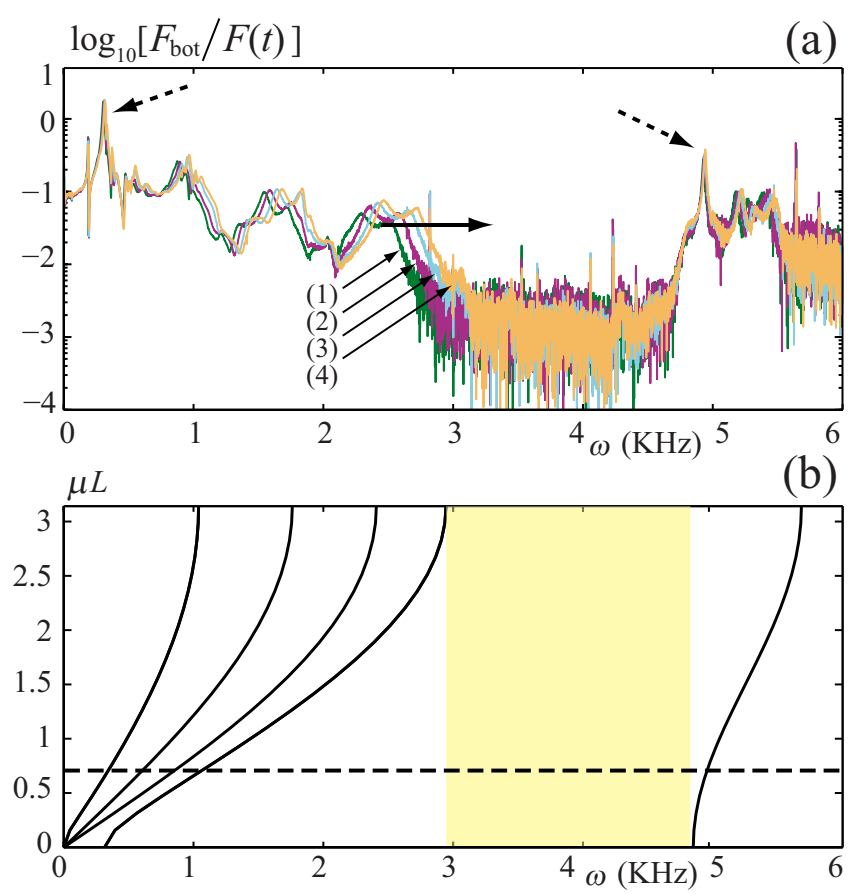

FIG. 2. (Color online) (a) Experimental base force $\left(F_{\text {bot }}\right)$ over input force $(F(t))$ as a function of frequency for four levels or precompression: line (1) $267 \mathrm{~N}$, line (2) $311 \mathrm{~N}$, line (3) $356 \mathrm{~N}$, and line (4) $400 \mathrm{~N}$. Solid arrow indicates band gap up shift for increasing precompression. Dashed arrows indicate resonant frequencies insensitive to precompression. (b) Dispersion relations obtained from FE model. The shaded area indicates a band gap. The dashed line indicates the wave number $(\mu L=0.71)$ used to evaluate the eigenmodes in Fig. 3.

dition to equilibrium conditions $f_{\text {top }}=-[I] e^{i \mu L} f_{\text {bot }}$ [Fig. 1(c)] in accordance with Bloch's theorem. ${ }^{11}$ Here, $[I]$ is the identity matrix and $\mu$ is the wave propagation constant. The resulting eigenvalue problem relates frequency $\omega$ and $\mu L{ }^{11}$

Load cells (PCB, $100 \mathrm{lbs}$ ) placed between the shaker and the specimen and between the specimen and the end plate [red blocks in Fig. 1(a)] recorded the force transmission for frequencies up to $6 \mathrm{KHz}$ [Fig. 2(a)]. The presence of an acoustic band gap is evident between 2.7 and $4.7 \mathrm{KHz}$ (in this frequency range the signal-to-noise ratio is expectedly very poor). As the precompression is increased from 267 to $400 \mathrm{~N}$, the acoustic band edge increases from $2505 \mathrm{~Hz}$ to $2730 \mathrm{~Hz}$ [solid arrow in Fig. 2(a)]. The behavior of the nonlinear crystal, however, is not tuned uniformly: two modes are unchanged when the precompression is increased [dashed arrows in Fig. 2(a)].
We performed a FE analysis to (i) confirm the presence of the acoustic band gap shown in Fig. 2(a) and (ii) investigate the response associated with the two frequency peaks insensitive to precompression. Our FE approach assumes that the o-rings were rigidly connected to steel disks and contact effects were not included (this assumption is valid as the static precompression is larger than the amplitude of dynamic deformations). Dispersion relations obtained from the FE model confirm the presence of a band gap between 3 and 4.7 KHz [Fig. 2(b)]. The wave number at which wave modes are inspected is chosen to approximately match the stationary frequency maxima of Fig. 2(a). The first mode [Fig. 3(a)] indicates shear-polarized waves in the $x y$-plane. This mode is not affected by static precompression, as its frequency is unaltered by the increasing static load [note the left dashed arrow in Fig. 2(a)]. On the contrary, the second, third, and fourth distinct wave modes are all sensitive to the applied precompression, as their frequency increases with increasing static load in Fig. 2(a). The second mode is characterized by rotations of the steel disks along the $z$-axis [orange arrows in Fig. 3(b)]. The third mode features axially polarized motion of the steel disks along the $z$-axis with most strain energy being confined in the o-rings [Fig. 3(c)]. The fourth mode is characterized by rotations of the disks in the $x$ or $y$-directions [Fig. 3(d)]. The fifth distinct mode is characterized by deformations of the steel disks, which store most strain energy [Fig. 3(e)]. Mechanical energy is then essentially propagated by a conveyor belt composed of resonating steel disks. This wave mode coincides with the upper band edge maximum in Fig. 2(a). Similarly to mode one, mode five is not sensitive to precompression. The sixth mode [Fig. 3(f)] also presents internal resonances with most strain energy being stored in the disks similarly to Fig. 3(e). This is typical of PCs composed of all solid elements with slender internal components. ${ }^{12}$

We also developed an analytical model to aide the design of future nonlinear PCs. The interaction of o-rings and disks obeys plane-strain conditions given the rotational symmetry. The relationship between load $P$ (per unit depth) and deformation $\delta$ (approach between disk and o-ring centers) for an infinitely long cylinder pressed against an elastic half space is $^{13}$

$$
\delta=P \frac{\left(1-\nu_{c}^{2}\right)}{\pi E^{*}}\left[2 \ln \left(2 d_{c} / a\right)-1\right],
$$

where the contact area $a^{2}=2 d_{c} P /\left(\pi E^{*}\right)$ and $E^{*}$ is the weighted stiffness. ${ }^{13}$ This relationship assumes a linearelastic behavior in contrast to previous analyses which as-

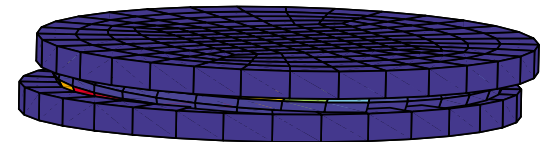

(a)

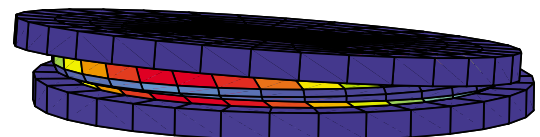

(d)

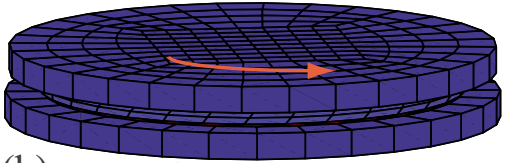

(b)

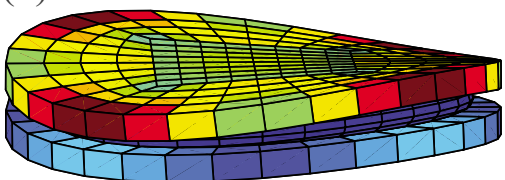

(e)

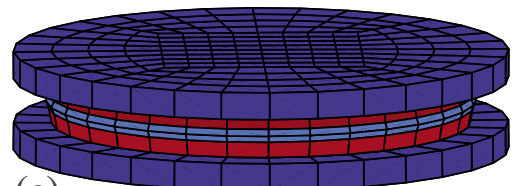

(c)

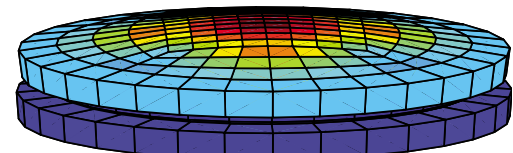

(f)

FIG. 3. (Color online) Wave modes associated with the dispersion relations of Fig. 2(b) and $\mu L=0.71$, for frequencies: (a) $343 \mathrm{~Hz}$, (b) $606 \mathrm{~Hz}$, (c) $854 \mathrm{~Hz}$, (d) $1063 \mathrm{~Hz}$, (e) $4951 \mathrm{~Hz}$, and (f) $8021 \mathrm{~Hz}$. 


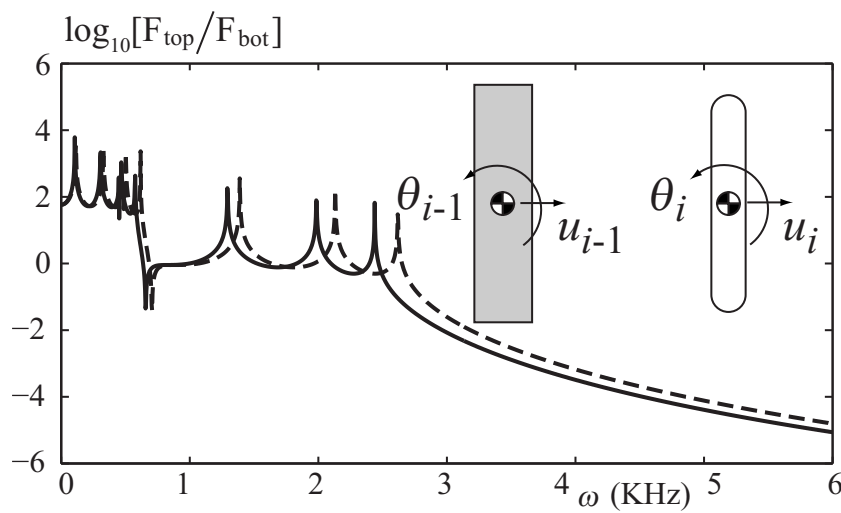

FIG. 4. Analytical force transmission for two values of initial precompression. Solid and dashed lines correspond to $267 \mathrm{~N}$ and $400 \mathrm{~N}$. The inset shows axial and rotational degrees of freedom included in the model.

sumed hyper-elastic behavior. ${ }^{10}$ The first order term of a Taylor expansion of Eq. (1) does not provide a linear relation between $P$ and $\delta$. A crude estimate of the linear stiffness needed to express $P=k_{L} \delta$ may be obtained simply as $k_{L}$ $=2[P / \delta]_{P=P_{0}}$ from Eq. (1). The factor 2 is needed as the o-rings are in contact with disks on top and bottom. The load transferred between o-rings and disks is then $P=2 \pi d_{0} k_{L} \delta$.

Load transducers recorded forces along the $z$-axis, which are associated with axial and rotational deformations. The axial $k_{L, a}$ and rotational $k_{L, r}$ stiffness for a precompression values of $267 \mathrm{~N}$ are evaluated as $k_{L, a}=138.8 \mathrm{MN}$ and $k_{L, r}$ $=12.2 \mathrm{KN} / \mathrm{rad}$. The same values for a precompression of 400 $\mathrm{N}$ are $k_{L, a}=160 \mathrm{MN}$ and $k_{L, r}=14.0 \mathrm{KN} / \mathrm{rad}$. The employed analytical model is a system of uncoupled, ordinary differential equations representing point masses connected by axial and rotational springs, solved assuming harmonic motion. The force transmission is evaluated for a sweep of frequencies (Fig. 4). These equations are $m_{i} \ddot{u}_{i}=k_{L, a}\left(u_{i-1}+u_{i+1}\right)$ $-2 k_{L, a} u_{i}$ for axial deformations and $I_{i} \ddot{\theta}_{i}=k_{L, r}\left(\theta_{i-1}+\theta_{i+1}\right)$ $-2 k_{L, r} \theta_{i}$ for rotations, where $m_{i}$ and $I_{i}$ are the mass and mass moment of inertia (inset in Fig. 4). This model qualitatively captures the dynamic response of the system when disks displace as rigid bodies [Figs. 3(a)-3(d)]. Disk resonance for $\omega>3 \mathrm{KHz}$ is not captured by this model.

In conclusion, we have designed and tested a onedimensional nonlinear PC whose multidimensional response is tailored via external precompression. A FE model is employed to confirm the existence of a significant band gap and to demonstrate that certain wave modes are not sensitive to precompression. Finally, an analytical model capable of estimating the acoustic band edge has been proposed to drive the design of future configurations.

We thank Allen Bronowicki. This work is part of a collaboration between Caltech and Northrop Grumman. C.D. acknowledges support from the Army Research Office (Grant No. 54272-EG, Dr. Bruce LaMattina) and the National Science Foundation (Grant No. 844540 CAREER).

${ }^{1}$ M. S. Kushwaha, P. Halevi, L. Dobrzynski, and B. Djafari-Rouhani, Phys. Rev. Lett. 71, 2022 (1993).

${ }^{2}$ X. Zhang and Z. Liu, Appl. Phys. Lett. 85, 341 (2004).

${ }^{3}$ S. Yang, J. H. Page, Z. Y. Liu, M. L. Cowan, C. T. Chan, and P. Sheng, Phys. Rev. Lett. 93, 024301 (2004).

${ }^{4}$ K. L. Jim, C. W. Leung, S. T. Lau, S. H. Choy, and H. L. W. Chan, Appl. Phys. Lett. 94, 193501 (2009).

${ }^{5}$ J. F. Robillard, O. Bou Matar, J. O. Vasseur, P. A. Deymier, M. Stippinger, A. C. Hladky Hennion, Y. Pennec, and B. Djafari-Rouhani, Appl. Phys. Lett. 95, 124104 (2009).

${ }^{6}$ K. Bertoldi and M. Boyce, Phys. Rev. B 77, 052105 (2008).

${ }^{7}$ V. F. Nesterenko, Dynamics of Heterogeneous Materials (Springer, New York, 2001)

${ }^{8}$ E. B. Herbold, J. Kim, V. F. Nesterenko, S. Wang, and C. Daraio, Acta Mech. 205, 85 (2009).

${ }^{9}$ I. I. L. D. Pinto, A. Rosas, A. H. Romero, and K. Lindenberg, Phys. Rev. E 82, 031308 (2010).

${ }^{10}$ E. B. Herbold and V. F. Nesterenko, Appl. Phys. Lett. 90, 261902 (2007).

${ }^{11}$ D. J. Mead, J. Sound Vib. 190, 495 (1996).

${ }^{12}$ A. Spadoni, M. Ruzzene, S. Gonella, and F. Scarpa, Wave Motion 46, 435 (2009).

${ }^{13}$ K. L. Johnson, Contact Mechanics (Cambridge University Press, Cambridge, 1987). 